\title{
ON SOME DETERMINANTS RELATED TO $p$-VALENT FUNCTIONS
}

\author{
BY \\ A. W. GOODMAN( $(1)$
}

1. Introduction. A function $f(z)$ is said to be $p$-valent in the open unit circle (hereafter denoted by $E$ ) if it is regular and assumes no value more than $p$ times $\left({ }^{2}\right)$ for $|z|<1$. The Bieberbach conjecture states that if

$$
w=f(z)=\sum_{n=1}^{\infty} b_{n} z^{n}
$$

is univalent in $E$, then $\left|b_{n}\right| \leqq n\left|b_{1}\right|$. This conjecture has been proved in many special cases and has a long history $\left(^{8}\right)$. To the best of our knowledge it has not been generalized to the class of $p$-valent functions. This is done in $\S 3$. In $\S 4$ it is shown that the truth of this conjecture would imply a set of trigonometric inequalities, Theorem 3 , which are generalizations of the elementary $|\sin n \theta / \sin \theta| \leqq n$. A proof of these inequalities is given in $\S 5$. Conversely it is shown, Theorem 6 , that these inequalities have an implication which tends to strengthen the conjecture slightly. Theorem 5 gives a second set of trigonometric inequalities which are generalizations of the trivial $|\cos n \theta| \leqq 1$. Finally in $\$ 6$ we note that the same methods may be used to obtain bounds for analogous algebraic expressions. This last result, Theorem 7 , is not new $\left({ }^{4}\right)$ but the method of proof is different.

2. Precise statements of theorems. A recent result $\left({ }^{5}\right)$ is:

Biernacki's Theorem. If $f(z)$, given by $(1.1)$, is p-valent in $E$ then

$$
\left|b_{n}\right| \leqq C(p) \mu_{p} n^{2 p-1}
$$

where

$$
\mu_{p}=\max \left\{\left|b_{1}\right|,\left|b_{2}\right|, \cdots,\left|b_{p}\right|\right\}
$$

and $C(p)$ is a function of $p$ alone.

Definition of $B_{n}^{*}$. Let $B_{1}, B_{2}, \cdots, B_{p}$ be a set of non-negative

Presented to the Society, August 22, 1946; received by the editors January 18, 1947.

(1) National Research Council Pre-doctoral Fellow.

(2) The usual definition of $p$-valence requires also that $f(z)$ assumes some value exactly $p$ times. For our purposes it is more convenient to consider a $q$-valent function as being also $p$-valent whenever $q \leqq p$.

(3) See for example Montel, Leçons sur les fonctions univalentes ou multivalentes, GauthierVillars, 1933, pp. 48-50.

(4) For historical notes and comments, see $\$ 6$.

(5) Biernacki, M., Sur les fonctions multivalentes d'ordre p, C. R. Acad. Sci. Paris vol. 203 (1936) pp. 449-451. See also Les fonctions multiralentes, Hermann et Cie., Editeurs, 1938. Actually Biernacki proved more then we have stated here. 
numbers. Let $\mathfrak{M}$ be the set of all functions of the form (1.1) which are $p$-valent in $E$ and for which

$$
\left|b_{k}\right| \leqq B_{k} \quad k=1,2, \cdots, p .
$$

The functions $B_{n}^{*}=B_{n}^{*}\left(B_{1}, B_{2}, \cdots, B_{p}, p\right), n>p$, are defined by the following two properties:

$(\alpha)$ If $f(z) \in \mathfrak{M}$, then $\left|b_{n}\right| \leqq B_{n}^{*}, n>p$, and

( $\beta) B_{n}^{*}$ is the smallest function for which $(\alpha)$ always holds.

Biernacki's theorem shows that the functions $B_{n}^{*}$ exist, that to bound the $n$th coefficient of a $p$-valent function it is sufficient to bound the first $p$ coefficients. Theorem 1 states roughly that it is also necessary.

TheOREM 1. Let $k$ be a fixed integer, $1 \leqq k \leqq p$. Let $B_{1}, B_{2}, \cdots, B_{k-1}$, $B_{k+1}, \cdots, B_{p}$ be any set of $p-1$ non-negative numbers. Let $M$ be assigned arbitrarily large. There exists a function $f_{M}(z)$ of the form (1.1) p-valent in $E$ and such that

$$
\left|b_{i}\right|=B_{j}, \quad i=1,2, \cdots, k-1, k+1, \cdots, p,
$$

and

$$
\left|b_{j}\right|>M, \quad i=p+1, p+2, \cdots
$$

THEOREM 2.

$$
B_{n}^{*} \geqq \sum_{k=1}^{p} B_{k} D(p, k, n), \quad n=p+1, p+2, \cdots
$$

where

$$
D(p, k, n)=\frac{2 k n \prod_{\alpha=1}^{p}\left(n^{2}-\alpha^{2}\right)}{(p+k) !(p-k) !\left(n^{2}-k^{2}\right)}, \quad 1 \leqq k \leqq p<n .
$$

The conjecture is that one actually has the equality sign in equation (2.5). Recently Yosida $\left({ }^{6}\right)$ has published a proof of this in the special case $B_{1}=B_{2}$ $=\cdots=B_{p-1}=0, n=p+1$.

THEOREM 3. Let

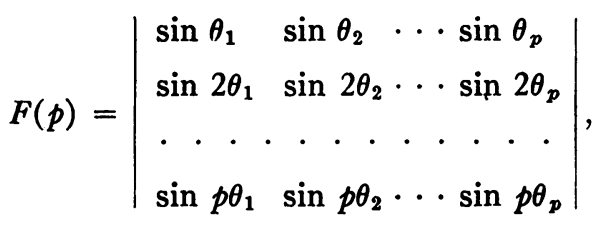

(9) Bemerkungen ibber die p-wertigen Funktionen, Proc. Imp. Acad. Tokyo vol. 20 (1944) pp. 16-19. 


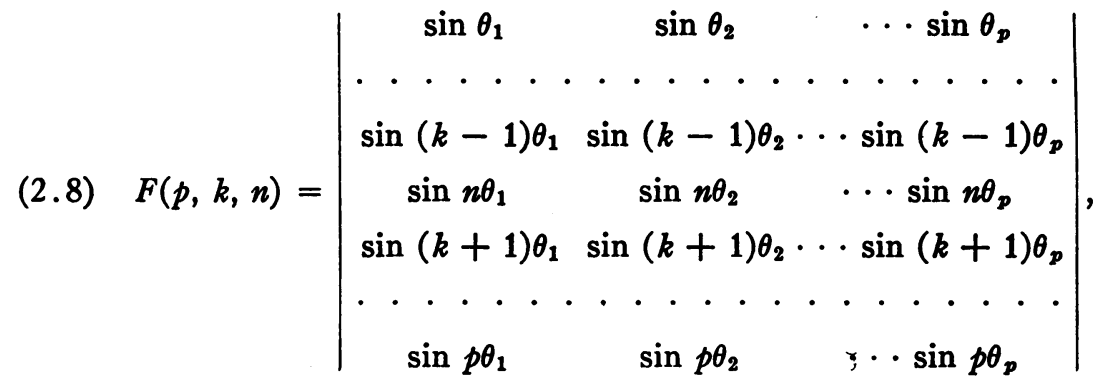

(2.9) $S(p, k, n)=F(p, k, n) / F(p)$.

If the $\theta_{j}$ are all real, then for all integers $n>p \geqq k \geqq 1$,

$$
|S(p, k, n)| \leqq D(p, k, n)
$$

with equality holding in the limit as all $\theta_{j} \rightarrow 0$.

THEOREM 4 The truth of the conjecture implies the truth of Theorem 3.

THEOREM 5. Let

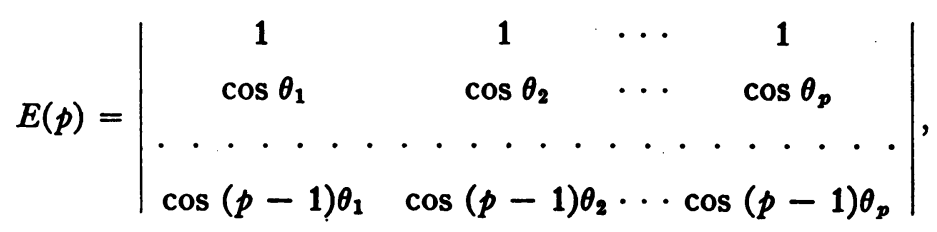

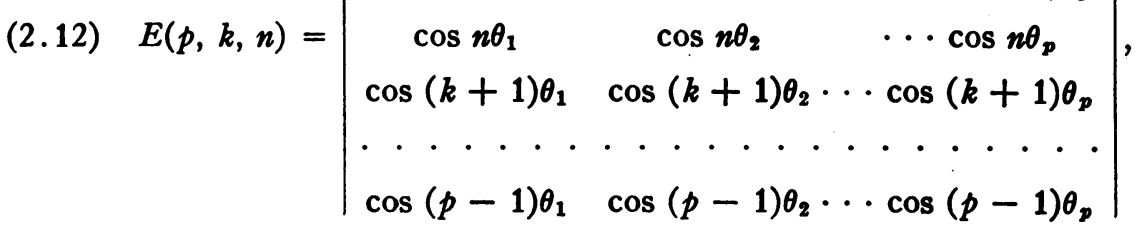

(2.13) $C(p, k, n)=E(p, k, n) / E(p)$.

If the $\theta_{j}$ are all real, then for all integers $n>p-1 \geqq k \geqq 1$,

$$
|C(p, k, n)| \leqq \frac{n}{k} D(p-1, k, n),
$$

$$
|C(p, 0, n)| \leqq \frac{\prod_{\alpha=1}^{p-1}\left(n^{2}-\alpha^{2}\right)}{(p-1) !(p-1) !}
$$


with equality holding in the limit as all $\boldsymbol{\theta}_{j} \rightarrow 0$.

THEOREM 6. Let $\{s(z)\}$ be the set of functions p-valent in $E$ of the form

$$
w=s(z)=P(u) / Q(u), \quad u=z /(1-z)^{2},
$$

where $P(u)$ and $Q(u)$ are polynomials of degree at most $p$. For this set of functions the conjecture holds.

Theorem 7 (Mitchell). Let

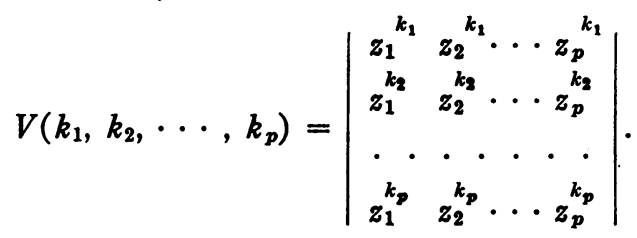

If $\left|z_{j}\right| \leqq 1, j=1,2, \cdots, p$, then for all integers $k_{p}>k_{p-1}>\cdots>k_{1} \geqq 0$

$$
\left|\frac{V\left(k_{1}, k_{2}, \cdots, k_{p}\right)}{V(0,1, \cdots, p-1)}\right| \leqq \frac{\prod_{h>i=1}^{p}\left(k_{h}-k_{j}\right)}{(p-1) !(p-2) ! \cdots 3 ! 2 !}
$$

with equality holding in the limit as all $z_{j} \rightarrow 1$.

LEMMA 1. For all integers $n \geqq k \geqq 1$

$$
\sum_{m=k}^{n} \frac{(-1)^{m+k} 2 n k}{(m+k) !(m-k) !} \prod_{\alpha=1}^{m-1}\left(n^{2}-\alpha^{2}\right)=\delta_{n}^{k},
$$

and for all integers $n>k \geqq 1, \beta \geqq 1$

$$
\sum_{m=k}^{n}(-1)^{m} \prod_{\alpha=1}^{m-1}\left(n^{\beta}-\alpha^{\beta}\right) \prod_{\alpha=m+1}^{n}\left(\alpha^{\beta}-k^{\beta}\right)=0 .
$$

Here the empty product is unity by definition.

3. The example functions. These functions are constructed by taking appropriate polynomials of a particular univalent function. This procedure will give the following lemma.

Lemma 2. The function (1.1) is p-valent in $E$ whenever

$$
b_{n}=\sum_{k=1}^{p}(-1)^{p-k} b_{k} D(p, k, n), \quad n=p+1, p+2, \cdots
$$

Theorem 1 follows immediately from Lemma 2 by selecting $b_{k}$ sufficiently large and $b_{j}=B_{j}, j=1,2, \cdots, k-1, k+1, \cdots, p$. Theorem 2 also follows from this lemma by setting $b_{k}=(-1)^{p-k} B_{k}, k=1,2, \cdots, p$.

To prove Lemma 2, observe first that 


$$
w=P(u)=a_{1} u+a_{2} u^{2}+\cdots+a_{p} u^{p}=\sum_{n=1}^{\infty} b_{n} z^{n}
$$

where $u=z /(1-z)^{2}=\sum_{n=1}^{\infty} n z^{n}$ is always $p$-valent in $E$. For $u(z)$ takes $E$ into the complex $u$-plane slit along the negative real axis from $-\infty$ to $-1 / 4$ and $w=P(u)$ maps this region into a Riemann surface having at most $p$ sheets. Now

$$
u^{m}=\sum_{n=1}^{\infty} A_{n}^{(m)} z^{n}, \quad \quad m=1,2, \cdots
$$

where

$$
A_{n}^{(m)}=\frac{(n+m-1)(n+m-2) \cdots(n-m+1)}{(2 m-1) !} .
$$

Using (3.3) in (3.2), it follows that

$$
b_{n}=\sum_{m=1}^{p} a_{m} A_{n}^{(m)}, \quad n=1,2, \cdots
$$

We shall determine the $a_{i}$ as functions of the $b_{j}, i, j=1,2, \cdots, p$, and from these determine the $b_{n}, n>p$, as functions of the $b_{j}, j=1,2, \cdots, p$. For this define $D^{\prime}(p, k, n)^{\prime}$ by

$$
D^{\prime}(p, k, n)=\left|\begin{array}{cccc}
A_{1}^{(1)} & A_{1}^{(2)} & \cdots & A_{1}^{(p)} \\
A_{2}^{(1)} & A_{2}^{(2)} & \cdots & A_{2}^{(p)} \\
\cdots & \cdots & \cdots & \cdot \\
A_{k-1}^{(1)} & A_{k-1}^{(2)} & \cdots & A_{k-1}^{(p)} \\
A_{n}^{(1)} & A_{n}^{(2)} & \cdots & A_{n}^{(p)} \\
A_{k+1}^{(1)} & A_{k+1}^{(2)} & \cdots & A_{k+1}^{(p)} \\
\cdots & \cdots & \cdots & \cdot \\
A_{p}^{(1)} & A_{p}^{(2)} & \cdots & A_{p}^{(p)}
\end{array}\right| .
$$

Now $n$ appears in only one row, and in each element of that row it occurs as a factor no more than $2 p-1$ times. So $D^{\prime}(p, k, n)$ is a polynomial in $n$ of degree at most $2 p-1$. For $n=j \neq k$ the $j$ th and $k$ th rows are identical and hence $D^{\prime}(p, k, n)$ has the roots $n=1,2, \cdots, k-1, k+1, \cdots, p$. Further the polynomial is an odd function of $n$. Finally for $n=0$ every element of the $k$ th row is zero. We thus know all the roots. To find the multiplicative constant for this polynomial observe that for $n=k$ every element above the main diagonal of (3.6) vanishes while each element in the main diagonal is one. An examination of (2.6) will show that 


$$
D^{\prime}(p, k, n)=(-1)^{p-k} D(p, k, n) .
$$

If now the first $p$ equations of the set (3.5) are solved for the $a_{i}$ in terms of the $b_{j}$, one finds that

$$
a_{m}=\sum_{k=1}^{m}(-1)^{k+m} b_{k} D(m-1, k, m)=\sum_{k=1}^{m}(-1)^{k+m} b_{k} \frac{2 k(2 m-1) !}{(m+k) !(m-k) !}
$$

for $1 \leqq m \leqq p$. Using (3.8) in (3.5)

$$
\begin{aligned}
b_{n} & =\sum_{m=1}^{p} A_{n}^{(m)} \sum_{k=1}^{m} b_{k}(-1)^{k+m} \frac{2 k(2 m-1) !}{(m+k) !(m-k) !} \\
& =\sum_{k=1}^{p} b_{k} \sum_{m=k}^{p} \frac{(-1)^{k+m} 2 k n}{(m+k) !(m-k) !} \prod_{\alpha=1}^{m-1}\left(n^{2}-\alpha^{2}\right) \\
& =\sum_{k=1}^{p} b_{k} Q(p, k, n)
\end{aligned}
$$

where the last equation defines $Q(p, k, n)$. Now (3.9) holds for any values assigned to $b_{1}, b_{2}, \cdots, b_{p}$. This establishes the identity (2.18), which is the first half of Lemma 1. Note that a slight change has been made in the upper limit of the sum which is permissible in view of the occurrence of zero factors. We shall not prove the second half of Lemma 1 since it is not needed in the present work. Its proof is similar to that of the first half with the one change that in place of $A_{n}^{(m)}$ given by (3.4) one uses the slightly more general

$$
\bar{A}_{n}^{(m)}=\frac{n \prod_{\alpha=1}^{m-1}\left(n^{\beta}-\alpha^{\beta}\right)}{m \prod_{\alpha=1}^{m-1}\left(m^{\beta}-\alpha^{\beta}\right)} .
$$

To obtain a simpler form for $Q(p, k, n)$ observe that it is a polynomial in $n$ of degree $2 p-1$, which by Lemma 1 has the roots $k+1, k+2, \cdots, p$ and the value one when $n=k$. Since all the terms of the sum are zero for $n=0,1,2, \cdots, k-1$, these values are also roots. Lastly $Q(p, k, n)$ is an odd function of $n$. Hence

$$
Q(p, k, n)=(-1)^{p-k} D(p, k, n)
$$

and using this in (3.9) we have (3.1). This completes the proof of Theorems 1 and 2.

4. A second set of $p$-valent functions. In $\S 3, p$-valent functions were obtained by taking pth degree polynomials of $u$. We next consider the rational functions $\left({ }^{7}\right)$ of $u, s(z)$ given by (2.15). These functions will be regular

( 7 ) The question of the size of the coefficients in the power series for functions of this form was first raised by O. Szász during a conversation in the autumn of 1945. 
in $E$ and hence $p$-valent in $E$ if and only if all the roots $\left(^{8}\right) u_{j}$ of $Q(u)$ lie in the interval $-\infty \leqq u_{j} \leqq-1 / 4$. We shall obtain power series for these functions and for this we need the following lemma.

Lemma $3\left({ }^{9}\right)$. Let $z_{j}=\cos \theta_{j}+i \sin \theta_{j}, j=1,2, \cdots, p$. Then

$$
\begin{aligned}
& E(p)=2^{(p-1)(p-2) / 2} \prod_{h>j=1}^{p}\left(\cos \theta_{h}-\cos \theta_{j}\right), \\
& E(p)=\left(\frac{1}{2}\right)^{p-1} \prod_{h>j=1}^{p}\left(z_{h}-z_{j}\right)\left(z_{h} z_{j}-1\right) \prod_{j=1}^{p} \bar{z}_{j}^{p-1}, \\
& F(p)=2^{p-1} E(p) \prod_{j=1}^{p} \sin \theta_{j}, \\
& F(p)=\left(\frac{1}{2 i}\right)^{p} \prod_{j=1}^{p}\left(z_{j}-\bar{z}_{j}\right) \bar{z}_{j}^{p-1} \prod_{h>j=1}^{p}\left(z_{h}-z_{j}\right)\left(z_{h} z_{j}-1\right) .
\end{aligned}
$$

If the indicated substitution is made, the trigonometric determinants are transformed into determinants which are algebraic in $z_{j}$ and $\bar{z}_{j}$. The relationship between $E(p)$ and $F(p)$ is then obvious. Finally a little manipulation of the rows will transform $E(p)$ into a determinant of Vandermonde type in the variables $\left(z_{j}-\bar{z}_{j}\right)$.

LeMma 4. Let $Q(u)$ be a fixed polynomial with all of its roots $u_{j}$ distinct and in the open interval $-\infty<u_{j}<-1 / 4$. Then there exists a unique function $s_{k}(z)$ of the set $\{s(z)\}$ of the form

$$
s_{k}(z)=z^{k}+\sum_{n=p+1}^{\infty} b_{n} z^{n}, \quad 1 \leqq k \leqq p,
$$

and for this function

$$
b_{n}=S(p, k, n), \quad n=p+1, p+2, \cdots,
$$

where the $\theta_{j}$ are such that

$$
-1<\cos \theta_{j}=1+1 / 2 u_{j}<1, \quad 0<\theta_{i}<\pi, \quad j=1,2, \cdots, p .
$$

Since the roots are assumed distinct we may write

$$
\begin{aligned}
& w=a_{0}+\sum_{j=1}^{p} \frac{a_{j} u}{u-u_{j}}=a_{0}+\sum_{j=1}^{p} \frac{a_{j} z}{z-u_{j}(1-z)^{2}}, \\
& w=a_{0}+\sum_{j=1}^{p} \frac{z A_{j} \sin \theta_{j}}{1-2 z \cos \theta_{j}+z^{2}}
\end{aligned}
$$

(8) When $Q(u)$ is of degree $q \leqq p$ we regard $p-q$ of the roots as being at $-\infty$.

(9) This was first obtained by E. Prouhet, Nouvelles Annales de Mathématiques (1) vol. 16 (1857) pp. 403-404 and vol. 17 (1858) pp. 187-190. 
where $\theta_{j}$ is determined as in (4.5) and $A_{j}=-a_{j} / u_{j} \sin \theta_{j}, j=1,2, \cdots, p$. For fixed $Q(u), P(u)$ determines (4.6) uniquely and conversely the $A_{j}$ of (4.6) determine $P(u)$ uniquely. It is well known $\left({ }^{10}\right)$ that

$$
\frac{z \sin \theta}{1-2 z \cos \theta+z^{2}}=\sum_{n=1}^{\infty} z^{n} \sin n \theta
$$

and so (4.6) becomes

$$
w=a_{0}+\sum_{n=1}^{\infty}\left(\sum_{j=1}^{p} A_{j} \sin n \theta_{j}\right) z^{n} .
$$

To obtain the form (4.3) from (4.8) take $a_{0}=0$, and determine the $A_{j}$ from the set of $p$ nonhomogeneous linear equations obtained by using the conditions on the first $p$ coefficients of the power series (4.3). The determinant of this system of equations is just $F(p)$, and our hypotheses on the roots $u_{j}$ together with (4.2) show that $F(p) \neq 0$. The $A_{j}$ are uniquely determined and using the values of $A_{j}$ found, it is easy to see that $b_{n}$ has the form (4.4).

It should be noted that in Lemma 4 the polynomial could be of degree less than $p$ and could have repeated roots subject only to the condition that all of the roots are in the interval $-\infty \leqq u_{j} \leqq-1 / 4$. Under these circumstances the quantities $S(p, k, n)$ become indeterminate forms, but the $b_{n}$ may still be obtained as limit values of the $S(p, k, n)$. To show this we denote by $Q_{1}(u)$ a polynomial of this slightly larger class. Without loss of generality one can always put $Q_{1}(u)$ in the form $1+c_{1} u+c_{2} u^{2}+\cdots+c_{p} u^{p}$ where, in view of the conditions imposed on the roots of $Q_{1}(u)$, the coefficients $c_{j}$ are bounded continuous functions of the roots. Then the $n$th coefficient in the Maclaurin series for $1 / Q_{1}(u)$ is also a bounded continuous function of the roots. Finally, $P(u)$ can always be determined, and uniquely so that $P(u) / Q_{1}(u)$ has the form (4.3), and when this is done each $b_{n}$ so obtained is a continuous bounded function of the roots of $Q_{1}(u)$. To complete the proof of the assertion about limits one only needs to select a sequence of polynomials $Q^{(v)}(u)$ which satisfy the conditions of Lemma 4 and such that $\lim _{\nu \rightarrow \infty} Q^{(v)}(u)=Q_{1}(u)$.

\section{LEMMA 5.}

$$
\lim _{\theta_{5} \rightarrow 0, j=1,2, \ldots, p} S(p, k, n)=(-1)^{p-k} D(p, k, n) .
$$

In the discussion above take $Q_{1}(u)=1$. Then (2.15) becomes (3.2). On the other hand $Q_{1}(u)$ is the limit of a sequence of polynomials whose roots $u_{j} \rightarrow \infty$ and so $\cos \theta_{j} \rightarrow 1$ and $\theta_{j} \rightarrow 0$. Lemma 5 then follows from equation (3.1). The proof of Theorem 4 is now obvious.

(10) Zygmund, Trigonometrical series, pp. 1-2. 
5. Proof of the trigonometric inequalities. We shall say that a polynomial has the sign $(-1)^{m}$ if the coefficient of every term of the polynomial has this sign. It is understood that this criterion is applied after like terms have been combined. For example $p(x)=3 x^{8}-2 x^{8}+x$ has the sign +1 . When $m$ is known to be even (odd) we shall merely say the polynomial is positive (negative).

The plan of proof for Theorems 3 and 5 is simple. It will be shown that the substitutions $z_{j}=\cos \theta_{j}+i \sin \theta_{j}, j=1,2, \cdots, p$, reduce $C(p, k, n)$ and $S(p, k, n)$ to polynomials in $z_{j}$ and $\bar{z}_{j}$ having a certain sign. Since, for real $\theta_{j},\left|z_{j}\right|=1$, it will follow that maximum absolute values for these polynomials occur when $z_{j}=\bar{z}_{j}=1$, or all $\theta_{j}=0$. Let

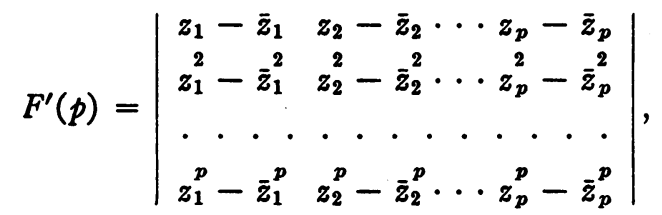

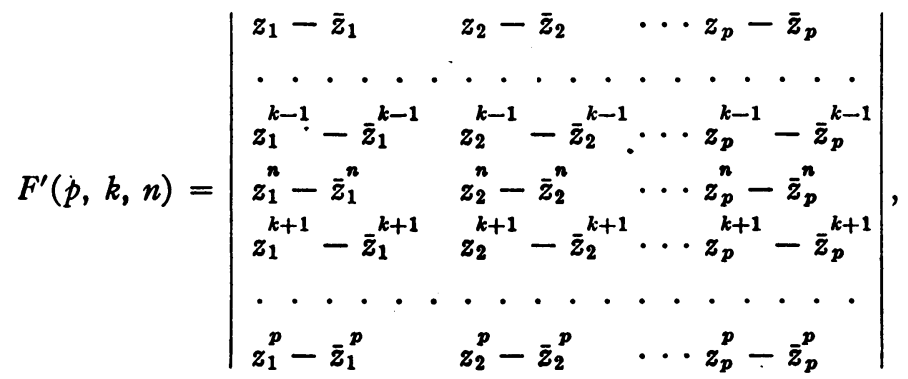

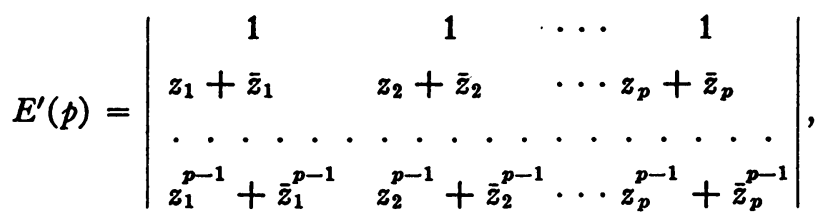

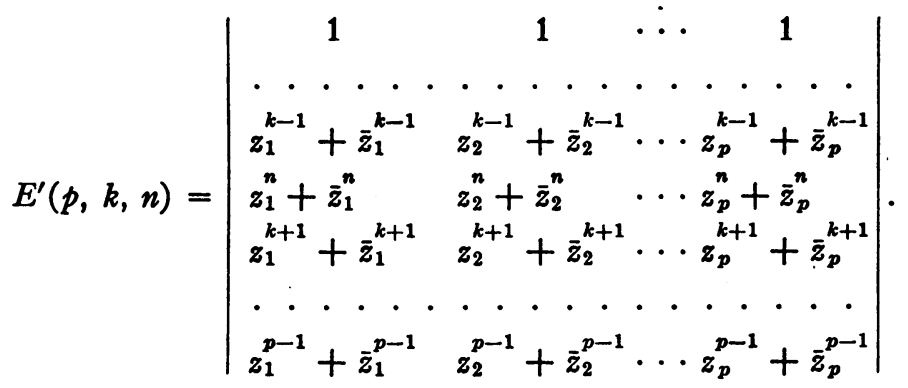

The following are trivial consequences of Lemma 3 and the above definitions 


$$
\begin{array}{rlrl}
C(p, k, n) & =E^{\prime}(p, k, n) / E^{\prime}(p), & 1 \leqq k \leqq p-1, \\
C(p, 0, n) & =2^{-1} E^{\prime}(p, 0, n) / E^{\prime}(p), & \\
S(p, k, n) & =F^{\prime}(p, k, n) / F^{\prime}(p), & 1 \leqq k \leqq p . \\
E^{\prime}(p)=2^{p-1} E(p) & =\prod_{n>j=1}^{p}\left(z_{h}-z_{j}\right)\left(z_{h} z_{j}-1\right) \prod_{1}^{p} \bar{z}_{j}^{p-1}, & \\
F^{\prime}(p)= & (2 i)^{p} F(p)=\prod_{h=1}^{p}\left(z_{h}-\dot{\bar{z}}_{h}\right) \prod_{h>j=1}^{p}\left(z_{h}-z_{j}\right)\left(z_{h} z_{j}-1\right) \prod_{1}^{p} \bar{z}_{j}^{p-1} .
\end{array}
$$

To avoid the annoying special treatment which arises in (5.5) when $k=0$, we introduce the definition $C^{\prime}(p, k, n)=E^{\prime}(p, k, n) / E^{\prime}(p), 0 \leqq k \leqq p-1$.

Lemma 6. For all integers $n>p-1 \geqq k \geqq 0, C^{\prime}(p, k, n)$ is a polynomial in $z_{j}$ and $\bar{z}_{j}, j=1,2, \cdots, p$, and this polynomial has the sign $(-1)^{p-k-1}$.

We use induction on $p$, the order of the determinant, which is valid for every admissible $k$ and $n$. For $p=1$ the theorem is trivial. Unfortunately the proof must be broken up into four cases according as $k=0,1 \leqq k \leqq p-3$, $k=p-2$, and $k=p-1$. Even this is not exhaustive since in each of these cases restrictions are necessarily imposed on the range of $p$. Certain special cases are thus omitted and must receive individual attention. A few new symbols will be of assistance. Let

$$
P_{\bullet}\left(z_{j}, z_{1}\right)=1+\sum_{\alpha=1}^{s}\left(z_{j} z_{1}\right)^{\alpha}+\left(\bar{z}_{j} z_{1}\right)^{\alpha}, \quad s=0,1,2, \cdots .
$$

Here, and in what follows, the empty sum is zero by definition. Then('11) for each $s \geqq 1$,

$$
\begin{aligned}
& \dot{z}_{j}+\dot{z}_{j}^{*}-\dot{z}_{1}-\bar{z}_{1}^{s}-z_{1}\left(z_{j}^{s-1}+\dot{z}_{j}^{s-1}-z_{1}^{s-1}-\dot{z}_{1}^{s-1}\right) \\
& =\bar{z}_{j} \bar{z}_{1}^{*}\left(z_{j} z_{1}-1\right)\left(z_{j}-z_{1}\right) P_{s-1}\left(z_{j}, z_{1}\right) \text {. }
\end{aligned}
$$

Further let

$$
\begin{array}{rr}
Q_{s}\left(z_{j}, z_{1}\right)=\left\{\sum_{\alpha=0}^{s}\left(z_{j} z_{1}\right)^{\alpha}\right\}\left\{\sum_{\alpha=0}^{s}\left(\bar{z}_{j} z_{1}\right)^{\alpha}\right\}, & s=0,1,2, \cdots, \\
K(s, m)=\sum_{\alpha=0}^{s-m} z_{1}^{m+2 \alpha}, & 0 \leqq m \leqq s .
\end{array}
$$

Then

$$
Q_{s}\left(z_{j}, z_{1}\right)=K(s, 0)+\sum_{m=1}^{s}\left(z_{j}^{m}+\bar{z}_{j}^{m}\right) K(s, m), \quad s \geqq 0,
$$

(II) It will be well to bear in mind that this identity as well as all those which follow depend on the assumption that $z_{j} \bar{z}_{j}=1, j=1,2, \cdots, p$. 


$$
\dot{z}_{j}^{\prime}+\dot{z}_{j}^{s}-\dot{z}_{1}^{s}-\dot{z}_{1}^{s}=\bar{z}_{j} \dot{z}_{1}^{\prime}\left(z_{j} z_{1}-1\right)\left(z_{j}-z_{1}\right) Q_{s-1}\left(z_{j}, z_{1}\right), \quad s \geqq 1 .
$$

Case $1 \leqq k \leqq p-3, p \geqq 4$. Perform the following transformations on $E^{\prime}(p, k, n)$, the numerator of $C^{\prime}(p, k, n):$ (1). Subtract the first column from the $j$ th column for $j=2,3, \cdots, p$. (2) Subtract $z_{1}$ times the $j$ th row from the $(j+1)$ th row for the values of $j$ in the order $j=p-1, p-2, \cdots, k+2$, $k-1, k-2, \cdots, 2,1$. If now the identities (5.8) and (5.12) are used, then certain obvious factors, common to either some row or some column, can be utilized to reduce the denominator $E^{\prime}(p)$ to $E^{\prime}(p-1)$ in the variables $z_{2}, z_{3}, \cdots, z_{p}$. Expansion of the numerator by minors of the first row yields only one nonzero term and so

(5.13) $C^{\prime}(p, k, n)=\frac{\overline{\bar{z}}_{1}^{(p-1)(p-2)+2 n-2 k / 2}}{E^{\prime}(p-1)}$

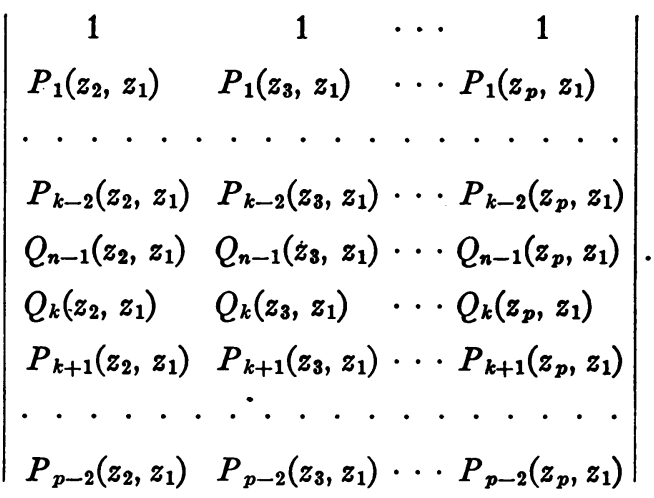

Now simple manipulations of the rows will reduce each $P_{s}\left(z_{j}, z_{1}\right)$ to the form $z_{1}^{s}\left(z_{j}^{s}+\bar{z}_{j}^{s}\right)$ except for $s=k+1$. In this case the simplification yields $\left.z_{1}^{k+1}\left(z_{j}^{k+1}+\bar{z}_{j}^{k+1}\right)+z_{1}^{k}\left(z_{j}^{k}+\bar{z}_{j}^{k}\right)+z_{1}^{k-1} z_{j}^{k-1}+\bar{z}_{j}^{k-1}\right)$ for a typical term of the $(k+2)$ th row. Since $K(s, m)$ contains only the variable $z_{1}$, it is clear that the $(k+1)$ th row can be so altered as to have $z_{1}^{k}\left(z_{j}^{k}+\bar{z}_{j}^{k}\right)+\left(z_{1}^{k-1}+z_{1}^{k+1}\right)\left(z_{j}^{k-1}+\bar{z}_{j}^{k-1}\right)$ for a typical term. Subtracting the $(k+1)$ th row from the $(k+2)$ th row and again removing certain obvious powers of $z_{1}$ from each row gives

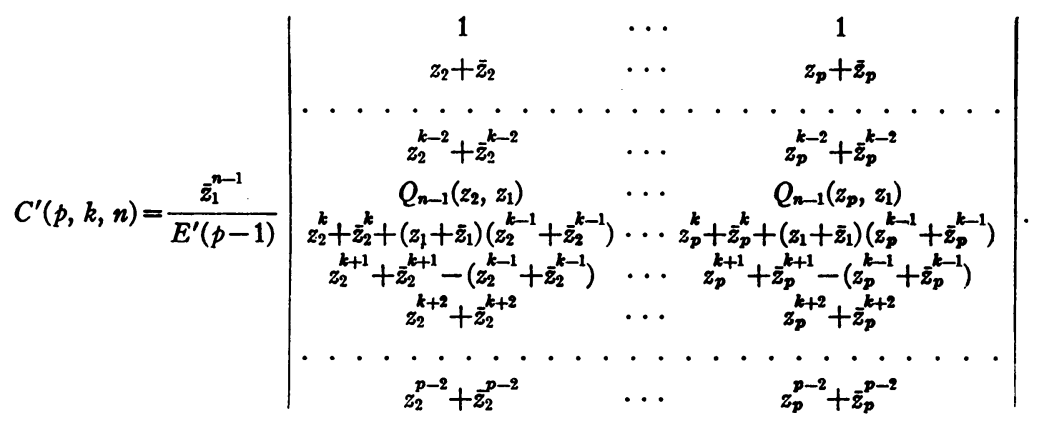

It should be noted that (5.14) is not strictly correct in the case $k=1$. It will be correct if wherever $z_{j}^{k-1}+\bar{z}_{j}^{k-1}$ occurs the value 1 is used in place of 2 . 
Using the $(k+1)$ th row and the $(k+2)$ th row $(5.14)$ can be expanded into the sum of four determinants one of which is obviously zero. Each of the three remaining derminants is again a sum of determinants when the form of $Q_{n-1}\left(z_{j}, z_{1}\right)$ as given by (5.11) is considered. When $1 \leqq m \leqq p-2$ in (5.11) most of the determinants are zero as a result of the equality of the $k$ th row with some other row. The terms, not obviously zero, obtained for $m \leqq p-2$ yield

$$
\bar{z}_{1}^{n-1}\left\{K(n-1, k-1)+K(n-1, k+1)-\left(z_{1}+\bar{z}_{1}\right) K(n-1, k)\right\}
$$

and if we use the definition (5.10) it is easy to see that this expression is identically zero. The terms obtained from $Q_{n-1}\left(z_{j}, z_{1}\right)$ when $m>p-2$ give

$$
\begin{aligned}
C^{\prime}(p, k, n)=\bar{z}_{1}^{n-1} \sum_{m=p-1}^{n-1} K(n-1, m)\left\{C^{\prime}(p-1, k-1, m)\right. \\
\left.\quad+C^{\prime}(p-1, k+1, m)-\left(z_{1}+\bar{z}_{1}\right) C^{\prime}(p-1, k, m)\right\} .
\end{aligned}
$$

If we use Lemma 6 for index $p-1$ it is clear that the polynomial (5.15) has the sign $(-1)^{p-k-1}$.

Case $k=p-2, p \geqq 4$. In this case the steps which led to (5.14) give

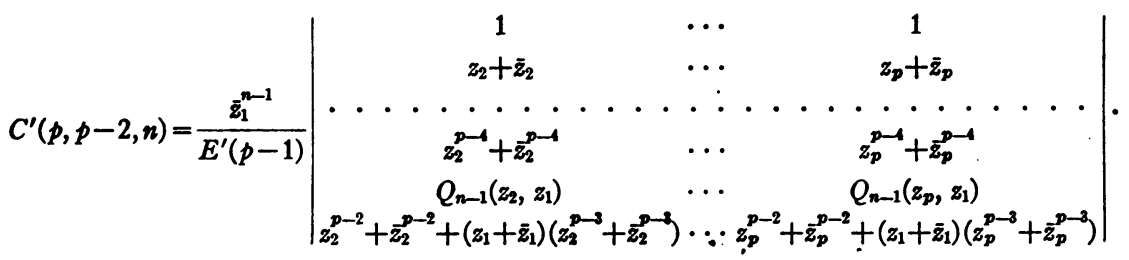

Using the last row (5.16) becomes the sum of two determinants. Each of these is again a sum of determinants when the form of $Q_{n-1}\left(z_{j}, z_{1}\right)$ is considered. For $1 \leqq m \leqq p-2$ most of the terms in this sum are zero. The nontrivial terms obtained for this range of $m$ yield

$$
\begin{array}{rlrl}
A & =\bar{z}_{1}^{n-1}\left\{K(n-1, p-3)-\left(z_{1}+\bar{z}_{1}\right) K(n-1, p-2)\right\} & \\
& =-\left(\bar{z}_{1}^{n-p}+\bar{z}_{1}^{n-p-2}+\cdots+z_{1}^{n-p-2}+z_{1}^{n-p}\right) & & (n>p) \\
& =-1 & & (n=p) .
\end{array}
$$

Combining this with the terms obtained when $m>p-2$ we have

$$
\begin{aligned}
C^{\prime}(p, p-2, n)=A+\bar{z}_{1}^{n-1} \sum_{m=p-1}^{n-1} K(n & -1, m)\left\{C^{\prime}(p-1, p-3, m)\right. \\
& \left.-\left(z_{1}+\bar{z}_{1}\right) C^{\prime}(p-1, p-2, m)\right\} .
\end{aligned}
$$

It is easy to see from this equation that the truth of Lemma 6 for index $p-1$ implies that $C^{\prime}(p, p-2, n)$ is a negative polynomial.

Case $k=p-1, p \geqq 3$. In this case the steps which before led to (5.14) give 
$(5.19)$

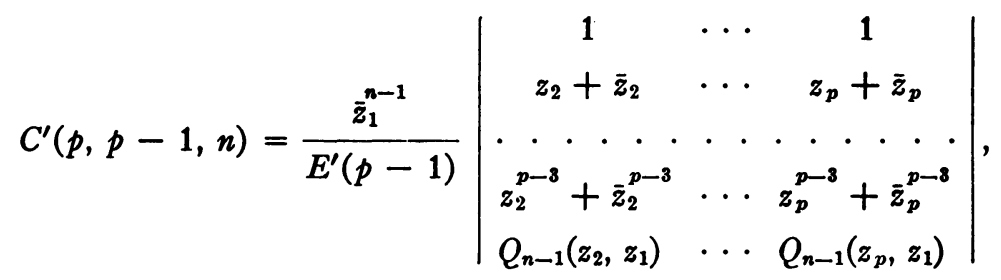

(5.20) $\quad C^{\prime}(p, p-1, n)=\bar{z}_{1}^{n-1} \sum_{m=p-2}^{n-1} K(n-1, m) C^{\prime}(p-1, p-2, m)$.

Case $k=0, p \geqq 3$. First more notation! Let

$$
R_{\bullet}\left(z_{j}, z_{1}\right)=\left(z_{j}^{s}+\bar{z}_{j}^{s}\right)\left(z_{1}+\bar{z}_{1}\right)-\left(z_{1}^{s}+\bar{z}_{1}^{s}\right)\left(z_{j}+\bar{z}_{j}\right), \quad s \geqq 1 .
$$

Then for $s \geqq 2$

$$
\begin{aligned}
R_{\varepsilon}\left(z_{j}, z_{1}\right)= & \bar{z}_{j}^{s} \dot{z}_{1}^{\prime}\left(z_{j} z_{1}-1\right)\left(z_{j}-z_{1}\right)\left\{\left(\sum_{\alpha=0}^{s} z_{j}^{\alpha \alpha} z_{1}^{\alpha}\right)\left(\sum_{\alpha=0}^{s-2} z_{j}^{\alpha-2-\alpha} z_{1}^{\alpha}\right)\right. \\
& \left.+\left(\sum_{\alpha=0}^{s-2} z_{j}^{\alpha \alpha} z_{1}^{\alpha}\right)\left(\sum_{\alpha=0}^{s} z_{j}^{s-\alpha} z_{1}^{\alpha}\right)\right\} .
\end{aligned}
$$

Let

$$
\begin{gathered}
T_{\theta}\left(z_{j}, z_{1}\right)=\bar{z}_{j}^{2}\left\{1+\sum_{\alpha=1}^{s} z_{j}^{\alpha \alpha} z_{1}^{\alpha}+\sum_{\alpha=1}^{s-2} \bar{z}_{j}^{\alpha} z_{1}^{\alpha}\right\}+1 \\
+\sum_{\alpha=1}^{s-2} z_{j}^{\alpha} z_{1}^{\alpha}+\sum_{\alpha=1}^{s} \bar{z}_{j}^{\alpha \alpha} z_{1}^{\alpha}
\end{gathered}
$$

Then for $s \geqq 2$

$$
\begin{aligned}
R_{\imath}\left(z_{j}, z_{1}\right)-z_{1} R_{\imath-1}\left(z_{j}, z_{1}\right) & =\bar{z}_{1}^{*}\left(z_{j} z_{1}-1\right)\left(z_{j}-z_{1}\right) T_{s}\left(z_{j}, z_{1}\right), \\
T_{\imath+1}\left(z_{j}, z_{1}\right)-T_{\imath}\left(z_{j}, z_{1}\right) & =\bar{z}_{j} z_{1}^{\prime}\left(z_{1}+\bar{z}_{1}\right)\left(z_{j}^{*}+\bar{z}_{j}^{*}\right) \\
T_{2}\left(z_{j}, z_{1}\right) & =\bar{z}_{j} z_{1}\left\{2+\left(z_{1}+\bar{z}_{1}\right)\left(z_{j}+\bar{z}_{j}\right)\right\} .
\end{aligned}
$$

Finally let

$$
U_{n}\left(z_{j}, z_{1}\right)=2 \sum_{m=1}^{n-1} z_{1}^{2 m}+z_{1}\left(z_{1}+\bar{z}_{1}\right) \sum_{m=1}^{n-1}\left(z_{j}^{m}+\bar{z}_{j}^{m}\right) K(n-1, m) .
$$

\section{Then for $n \geqq 2$}

$$
R_{n}\left(z_{j}, z_{1}\right)=\bar{z}_{j} \bar{z}_{1}^{n+1}\left(z_{j} z_{1}-1\right)\left(z_{j}-z_{1}\right) U_{n}\left(z_{j}, z_{1}\right) .
$$

In $E^{\prime}(p, 0, n)$ multiply each column except the first by $z_{1}+\bar{z}_{1}$. Then subtract from the $k$ th column $z_{k}+\bar{z}_{k}$ times the first column, for $k=2,3, \cdots, p$. Expansion by minors of the second row gives 


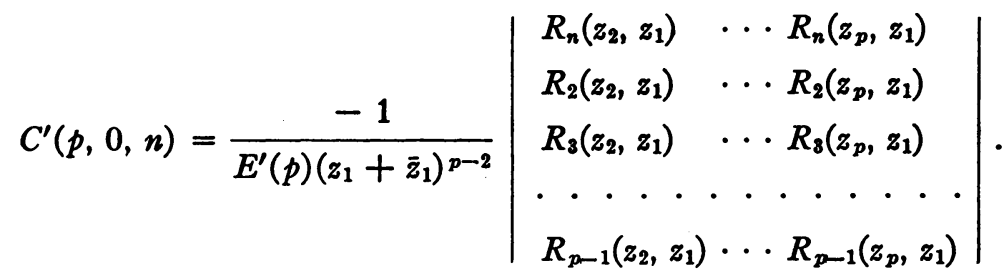

Next multiply the $(s-1)$ th row by $z_{1}$ and subtract the result from the $s$ th row for the values of $s$ in the order $s=p-1, p-2, \cdots, 4,3$. Removing obvious factors yields

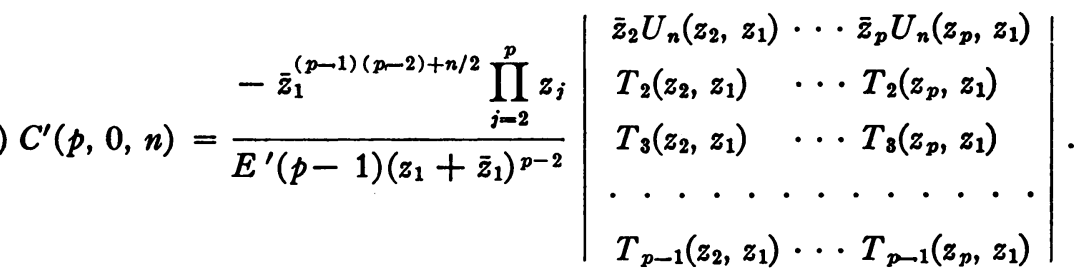

Again subtract the $s$ th row from the $(s+1)$ th row for the values of $s$ in the order $s=p-2, p-3, \cdots, 3,2$, and remove obvious factors. Then one finds

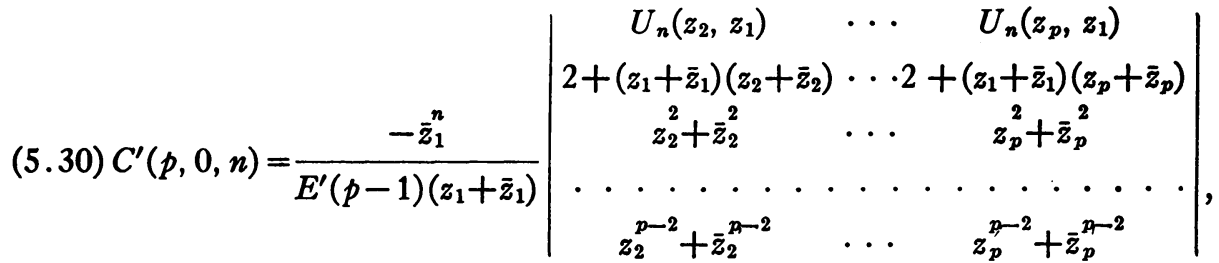

and since the expression $\bar{z}_{1}^{n}\left\{2 \sum_{m=1}^{n-1} z_{1}^{2 m}-2 z_{1} K(n-1,1)\right\}$ vanishes identically,

$$
\begin{aligned}
C^{\prime}(p, 0, n)=\bar{z}_{1}^{n-1} \sum_{m=p-1}^{n-1} K(n-1, m)\left\{2 C^{\prime}(p-1,1, m)\right. & \\
& \left.-\left(z_{1}+\bar{z}_{1}\right) C^{\prime}(p-1,0, m)\right\} .
\end{aligned}
$$

The inductive hypothesis applied to (5.31) shows that $C^{\prime}(p, 0, n)$ has the sign $(-1)^{p-1}$.

The above treatment omits the cases $p=2$, and $p=3, k=1$. After what has been done, these will present no new difficulties and so may be left for the reader.

Lemma 7. For all integers $n>p \geqq 1$

$$
\begin{array}{r}
S(p, k, n)=\sum_{\alpha=0}^{t} C^{\prime}(p, k-1, n-1-2 \alpha)-C^{\prime}(p, k+1, n-1-2 \alpha), \\
1 \leqq k \leqq p-2,
\end{array}
$$




$$
\begin{aligned}
S(p, k, n)=\sum_{\alpha=0}^{t} C^{\prime}(p, k-1, n-1-2 \alpha)+2^{-1}\left\{1+(-1)^{n-k}\right\}, \\
1 \leqq k=p-1, p,
\end{aligned}
$$

where $t=[(n-p-1) / 2]$.

This reduces the sine determinants to sums of cosine determinants. The computations are direct and will not be given.

Lemma 8. $S(p, k, n)$ is a polynomial in $z_{j}$ and $\bar{z}_{j}, j=1,2, \cdots, p$, having the sign $(-1)^{p-k}$.

It is clear that Lemma 8 can be proved from Lemmas 6 and 7 , if we group the terms in the sums in a proper manner. The next two lemmas do exactly this.

Lemma 9. $C^{\prime}(p, p-2, p)+1$ is a negative polynomial.

This is a trivial consequence of Lemma 6 taken together with the reduction formulae (5.18) and (5.17), when $p \geqq 4$. For $p=2,3$ individual computations are necessary.

LEMma 10. For all integers $n-1>p-2 \geqq k \geqq 1$

$$
C^{\prime}(p, k-1, n)-C^{\prime}(p, k+1, n)
$$

is a polynomial having the sign $(-1)^{p-k}$.

This is proved by induction on $p$, the order of the determinant, using the reduction identities already developed for the proof of Lemma 6 . Here again we are forced to consider cases.

Case $2 \leqq k \leqq p-4, p \geqq 6$. For these ranges of the indices both terms of (5.34) can be reduced by (5.15). Regrouping the terms, we have

$$
C^{\prime}(p, k-1, n)-C^{\prime}(p, k+1, n)=\bar{z}_{1}^{n-1} \sum_{m=p-1}^{n-1} K(n-1, m) \Delta_{1}
$$

where $\Delta_{1}=C^{\prime}(p-1, k-2, m)-C^{\prime}(p-1, k, m)+C^{\prime}(p-1, k, m)-C^{\prime}(p-1$, $k+2, m)-\left(z_{1}+\bar{z}_{1}\right)\left\{C^{\prime}(p-1, k-1, m)-C^{\prime}(p-1, k+1, m)\right\}$. Clearly the assertion for index $p$ follows in this case from the assumption of the lemma for index $p-1$.

Case $2 \leqq k=p-3, p \geqq 5$. Here (5.34) is reduced by using (5.15) on the first term and (5.18) on the second term. So

$$
C^{\prime}(p, p-4, n)-C^{\prime}(p, p-2, n)=-A+\bar{z}_{1}^{n-1} \sum_{m=p-1}^{n-1} K(n-1, m) \Delta_{2}
$$

where 


$$
\begin{aligned}
\Delta_{2}= & C^{\prime}(p-1, p-5, m)-C^{\prime}(p-1, p-3, m) \\
& -\left(z_{1}+\bar{z}_{1}\right)\left\{C^{\prime}(p-1, p-4, m)-C^{\prime}(p-1, p-2, m)\right\} \\
& +C^{\prime}(p-1, p-3, m) .
\end{aligned}
$$

The assumption of the lemma for index $p-1$ shows that the first four terms of $\Delta_{2}$ are so grouped as to be negative. The fifth term is also negative but the $-A$ is positive. If we apply Lemma 9 we see that for $m=p-1$ the fifth term contains -1 and so we have present from this term

$$
-\bar{z}_{1}^{n-1} K(n-1, p-1)=-\left(\bar{z}_{1}^{n-p}+\bar{z}_{1}^{n-p-2}+\cdots+z_{1}^{n-p-2}+z_{1}^{n-p}\right)=A .
$$

Thus (5.36) is a negative polynomial.

Case $2 \leqq k=p-2, p \geqq 4$. Here (5.34) is reduced by using (5.15) on the first term and (5.20) on the second term. Orserve that the range of summation is slightly different in the two formulae, and that the value $k=p-3$ used in (5.15) makes the second term of this formula identical with the term obtained from (5.20) when $k=p-1$. Thus

$$
\begin{aligned}
C^{\prime}(p, p-3, n) & -C^{\prime}(p, p-1, n) \\
& =-\bar{z}_{1}^{n-1} K(n-1, p-2)+\bar{z}_{1}^{n-1} \sum_{m=p-1}^{n-1} K(n-1, m) \Delta_{3}
\end{aligned}
$$

where $\Delta_{3}=C^{\prime}(p-1, p-4, m)-\left(z_{1}+\bar{z}_{1}\right) C^{\prime}(p-1, p-3, m)$. Now each of the terms of $\Delta_{3}$ is positive, and further by Lemma 9 we know that the second term of $\Delta_{3}$ contains -1 for $m=p-1$. So we have present from this term $\bar{z}_{1}^{n-1} K(n-1, p-1)\left(z_{1}+\bar{z}_{1}\right)$. All that remains is to observe that

$$
\bar{z}_{1}^{n-1} K(n-1, p-1)\left(z_{1}+\bar{z}_{1}\right)-\bar{z}_{1}^{n-1} K(n-1, p-2)
$$

is positive. Whence (5.37) is positive.

Case $1=k \leqq p-4, p \geqq 5$. Here (5.34) is reduced by using (5.31) on the first term and (5.15) on the second term. Thus

$$
C^{\prime}(p, 0, n)-C^{\prime}(p, 2, n)=\bar{z}_{1}^{n-1} \sum_{m=p-1}^{n-1} K(n-1, m) \Delta_{4}
$$

where $\Delta_{4}=C^{\prime}(p-1,1, m)-C^{\prime}(p-1,3, m)-\left(z_{1}+\bar{z}_{1}\right)\left\{C^{\prime}(p-1,0, m)\right.$ $\left.-C^{\prime}(p-1,2, m)\right\}$. It is clear from the grouping of terms indicated above that (5.39) has the sign $(-1)^{p-1}$.

The only cases which have been omitted in the above work are the cases $k=1, p=3,4$. These are relatively simple and will be left to the reader. This completes the proof of Lemma 10 and hence Lemma 8. Combining this with Lemma 5, Theorem 3 is established. 
In the same way Lemma 6 will give Theorem 5 as soon as we obtain the limit value of $C^{\prime}(p, k, n)$ as $z_{j} \rightarrow 1$. To do this we return to the trigonometric form and use L'Hospital's rule on $C(p, k, n)$. First let $k>0$. Set $\theta_{1}=0$. Now as $\theta_{2} \rightarrow 0$, the ratio becomes indeterminate and so we differentiate numerator and denominator with respect to $\theta_{2}$ just once. Now as $\theta_{3} \rightarrow 0$ we again have an indeterminate form and again we differentiate numerator and denominator, this time with respect to $\theta_{3}$. Proceeding in this way it is clear that

$$
\begin{aligned}
\lim _{\theta_{j} \rightarrow 0, j=1,2, \cdots, p} C(p, k, n) & =\frac{n}{k} \lim _{\theta_{j \rightarrow 0} \rightarrow j=2,3, \cdots, p} S(p-1, k, n) \\
& =\frac{n}{k}(-1)^{p-k-1} D(p-1, k, n) .
\end{aligned}
$$

When $k=0$ the treatment is a little different. Put $\theta_{1}=0$ and differentiate numerator and denominator once with respect to $\theta_{j}, j=2,3, \cdots, p$. The denominator is now reduced to a determinant of order $p-1$, but the numerator is still of $p$ th order. Expanding the numerator by minors of the first column, one has

$$
\begin{aligned}
\lim _{\theta_{j \rightarrow 0, j=1,2}, \ldots, p} C(p, 0, n) & =1-\lim _{\theta_{j} \rightarrow 0, j=2,3, \cdots, p} \sum_{k=1}^{p-1} \frac{n}{k} S(p-1, k, n) \\
& =1-\sum_{k=1}^{p-1} \frac{(-1)^{p-k-1} 2 n^{2} \prod_{\alpha=1}^{p-1}\left(n^{2}-\alpha^{2}\right)}{(p+k-1) !(p-k-1) !\left(n^{2}-k^{2}\right)} .
\end{aligned}
$$

The right side is an even polynomial in $n$ of degree $2 p-2$ with the obvious roots $\pm 1, \pm 2, \cdots, \pm(p-1)$ and the value 1 for $n=0$. So (5.40) and (5.41) yield (2.14) and this completes, the proof of Theorem 5 .

Theorem 6 is now an easy consequence of Theorem 3 and Lemma 4.

6. Proof of Mitchell's theorem(12). This theorem does not seem to be too well known and it is hoped that the appearance of the present proof will serve to call attention to it. As recently as 1938 Biernacki $\left({ }^{13}\right)$ refers to the simplest case $\left({ }^{14}\right)$ of this theorem as Itahara's lemma.

It has long been known that when the non-negative integers $k_{j}$ are arranged in an increasing sequence, $V\left(k_{1}, k_{2}, \cdots, k_{p}\right) / V(0,1, \cdots, p-1)$ is a positive polynomial $\left({ }^{12}\right)\left({ }^{15}\right)$. From this it follows immediately that

(12) O. H. Mitchell, Note on determinants of powers, Amer. J. Math. vol. 4 (1881) pp. 341-344.

(13) Les fonctions multivalentes, p. 10.

(14) The case $k_{p}=n, k_{j}=j-1, j=1,2, \cdots, p-1$. On the multivalency of power series, Jap. J. Math. vol. 10 (1933) pp. 71-78.

(15) P. C. Rosenbloom, Some properties of absolutely monotonic functions, Bull. Amer. Math. Soc. vol. 52 (1946) pp. 458-462. 


$$
\lim _{s_{j \rightarrow 1, j=1,2}, \cdots, p} \frac{V\left(k_{1}, k_{2}, \cdots, k_{p}\right)}{V\left(m_{1}, m_{2}, \cdots, m_{p}\right)}
$$

always exists whenever the integers $m_{j}$ are all distinct. We can determine the limit value by first considering the special case in which $m_{j}=k_{j}, j=1,2, \cdots$, $p-1$, and then combining these special cases in a rather obvious fashion. Consider the set of functions of the form

$$
w=\sum_{j=1}^{p} \frac{c_{j}}{1-z_{j} z}=\sum_{n=0}^{\infty} \sum_{j=1}^{p} c_{j} z_{j}^{n} z^{n}=\sum_{n=0}^{\infty} b_{n} z^{n}
$$

and, for a given fixed set of indices $0=k_{1}, k_{2}, \cdots, k_{p}$, select that function of the set for which

$$
\begin{aligned}
& b_{k_{j}}=0, \quad j=1,2, \cdots, p-1, \\
& b_{k_{p}}=1 .
\end{aligned}
$$

It is assumed of course that the $z_{j}$ are such that $V\left(k_{1}, k_{2}, \cdots, k_{p}\right) \neq 0$. The $c_{j}$ are then uniquely determined and using the expressions obtained for them by solving the equation set (6.3) in (6.2), one finds that

$$
w=\sum_{n=0}^{\infty} \frac{V\left(k_{1}, k_{2}, \cdots, k_{p-1}, n\right)}{V\left(k_{1}, k_{2}, \cdots, k_{p}\right)} z^{n}
$$

On the other hand the limit function as $z_{5} \rightarrow 1$ in (6.2) can be obtained by examining the set of functions

$$
\begin{aligned}
w & =\frac{d_{0}+d_{1} z+\cdots+d_{p-1} z^{p-1}}{(1-z)^{p}} \\
& =\frac{1}{z}\left(a_{1} v+a_{2} v^{2}+\cdots+a_{p} v^{p}\right)=\sum_{n=0}^{\infty} b_{n}^{\prime} z^{n},
\end{aligned}
$$

where $v=z /(1-z)$. Without too much difficulty it can be seen that the conditions (6.3) applied to (6.5) give

$$
b_{n}^{\prime}=\prod_{j=1}^{p-1}\left(n-k_{j}\right) / \prod_{j=1}^{p-1}\left(k_{p}-k_{j}\right) .
$$

Therefore the limit values of the coefficients of (6.4) are given by (6.6).

Finally we observe that the left side of $(2.17)$ can be obtained as a product of a finite number of terms of the form (6.1), and when this is done and the limit taken as $z_{5} \rightarrow 1$, the corresponding product of terms of the form (6.6) will yield the right side of (2.17). This completes the proof of Theorem 7 .

Columbia University,

NEW YORK, N. Y. 